\title{
The role of vaginal hysterectomy in the treatment of endometrial carcinoma
}

\author{
R. J. LELLÉ* ${ }^{*}$ G. W. MORLEY* \& W. A. PETERS ${ }^{\dagger}$ \\ ${ }^{*}$ Department of Obstetrics and Gynecology, University of Michigan Medical Center, Ann Arbor, Michigan and $\dagger$ Puget \\ Sound Oncology Consortium, Seattle, Washington, USA
}

\begin{abstract}
Lellé RJ, Morley GW, Peters WA. The role of vaginal hysterectomy in the treatment of endometrial carcinoma. Int J Gynecol Cancer 1994; 4: 342-347.
\end{abstract}

Between 1964 and 1991, vaginal hysterectomy was performed in 60 patients with clinical stage I endometrial carcinoma, who were not considered candidates for the conventional surgical approach. Of these patients, $66.7 \%$ were obese with a median weight of 235 pounds. Other risk factors included hypertension $(63 \%)$, diabetes mellitus (34\%), cardiac disease $(\mathbf{2 8 \%})$ and pulmonary disease $(\mathbf{1 2} \%)$. Operative mortality was $0 \%$. The complication rate was $14 \%$, with four patients requiring transfusions and four patients developing vaginal cuff cellulitis. Forty per cent of patients received adjuvant pre- or postoperative radiation therapy. Crude survival at 5 and 10 years was $91.1 \%$ and $87.1 \%$, respectively. However, only one patient died from disease 6 years after primary treatment. Although we consider surgical staging as the standard of care for the treatment of endometrial cancer, vaginal hysterectomy has a definite place in the management of patients with good prognostic criteria who are at high operative risk for the standard surgical approach.

KEYWORDS: complications, endometrial cancer, risk factors, survival, vaginal hysterectomy.

Vaginal hysterectomy used to be the treatment of choice for endometrial carcinoma in Europe ${ }^{(1-3)}$ as well as an acceptable treatment option in the USA ${ }^{(4)}$. Today, however, the attitude towards the vaginal approach has changed dramatically, and surgery through the abdominal route is considered the standard of care. This is illustrated by the fact that, in a recently published textbook, Park et al. ${ }^{(5)}$ do not discuss vaginal hysterectomy as a treatment option for patients with endometrial cancer.

In 1983, Peters et al. ${ }^{(6)}$ reported their experience with the use of vaginal hysterectomy in the treatment of endometrial carcinoma at the University of Michigan Medical Center and at the University of Virginia

Address for correspondence: Dr G. W. Morley, Department of Obstetrics and Gynecology, University of Michigan Medical Center, Room D2255, MPB 0718, 1500 East Medical Center Drive, Ann Arbor, MI 48109-0718, USA.
School of Medicine, USA. At the time of Peters' report, staging of endometrial cancer was done clinically, and abdominal hysterectomy and bilateral salpingooophorectomy with or without postoperative radiation was considered the treatment of choice for endometrial cancer. In 1988, the FIGO adopted surgical staging for carcinoma of the endometrium ${ }^{(7)}$, and peritoneal cytology as well as pelvic and paraaortic lymph node sampling became part of routine treatment. Consequently, if vaginal hysterectomy is to be considered as an alternative treatment, strict selection criteria must be applied, and treatment results have to be surveyed critically.

The following report represents our experience with 60 patients at the University of Michigan Medical Center, USA. It encompasses and updates the previously published data. In addition, 24 new patients are included in the study. It is the intention of the authors to revive the discussion of vaginal 
hysterectomy as treatment for endometrial cancer and to demonstrate that it is an acceptable treatment alternative for patients who are not candidates for standard abdominal surgery.

\section{Patients and methods}

Through the University of Michigan Tumor Registry, records of all patients were obtained who had been treated by vaginal hysterectomy for endometrial carcinoma between 1 January 1964 and 31 December 1991. Patients who underwent vaginal hysterectomy at an outside institution and were referred to the University of Michigan for further therapy were not included. Results from patients treated between 1964 and 1981 had been reported previously by Peters et $a l .{ }^{(6)}$ All charts were reviewed with regards to the medical history at the time of presentation, the treatment and hospital course as well as visits at the Gynecologic Tumor Clinic. Further follow-up data were obtained through the Michigan Tumor Registry. If outside slides were available, they were reviewed by a gynecologic pathologist at the University of Michigan. Statistical analysis was performed using the SPSSX program.

\section{Results}

Between 1964 and 1991, vaginal hysterectomy for endometrial cancer was performed on 60 patients. One to six patients were treated by vaginal surgery every year.

The clinical characteristics of the patients are summarized in Table 1 . The median age was 59.5 years. The youngest patient treated was 27 years old and the oldest patient 82 years. The median parity was three and the median weight 235 pounds, with a range of 118-422 pounds. The majority of patients had numerous medical problems. Hypertension (63\%),

Table 1. Patient characteristics

\begin{tabular}{|c|c|c|}
\hline Median age & 59.5 years & (27-82 years) \\
\hline Median parity & 3 & $(0-12)$ \\
\hline Median weight & 235 pounds & (118-422 pounds) \\
\hline Additional diagnoses & $n$ & $\%$ \\
\hline Hypertension & 38 & 63 \\
\hline Diabetes mellitus & 20 & 34 \\
\hline Cardiac disease & 17 & 28 \\
\hline Pulmonary disease & 7 & 12 \\
\hline \multicolumn{3}{|l|}{ Symptomatic pelvic } \\
\hline relaxation & 10 & 17 \\
\hline
\end{tabular}

diabetes mellitus (34\%) and cardiac disease (28\%) were the most frequent diagnoses. Furthermore, symptomatic pelvic relaxation was present in 10 patients $(17 \%)$.

As the abdominal approach was considered the standard of care for the time frame studied, the patients' charts state the specific indications for the vaginal surgery. These indications are listed in Table 2. Two-thirds of the patients were considered obese and, in $31.7 \%$ of cases, morbid obesity was the predominant determinant for the vaginal surgery. Frequently, the indication was based on the presence of several risk factors for abdominal surgery, including obesity, diabetes mellitus and cardiac disease.

Table 2. Indications for vaginal hysterectomy

\begin{tabular}{lrr}
\hline & $n$ & $\%$ \\
\hline Obese patients & 40 & 66.7 \\
$\quad$ Morbid obesity alone & 19 & 31.7 \\
Diabetes mellitus & 7 & 11.7 \\
Cardiac disease & 5 & 8.3 \\
Cardiac disease and diabetes & 3 & 5.0 \\
Pelvic relaxation & 3 & 5.0 \\
Sarcoidosis with renal damage & 1 & 1.7 \\
Recurrent ventral hernia & 1 & 1.7 \\
Previous anesthesia complication & 1 & 1.7 \\
& & \\
Non-obese patients & 20 & 33.3 \\
Premalignant lesion on D\&C & 6 & 10.0 \\
Pelvic relaxation and unexpected or & & \\
$\quad$ early invasive disease & 5 & 8.3 \\
Early invasive disease & 2 & 3.3 \\
Severe cardiac disease & 2 & 3.3 \\
Diabetes and pelvic relaxation & 1 & 1.7 \\
Parkinson's and severe cardiac disease & 1 & 1.7 \\
Liver metastasis from other primary & 1 & 1.7 \\
Indication not mentioned & 2 & 3.3 \\
\hline
\end{tabular}

Twenty patients (33.3\%) undergoing vaginal hysterectomy for endometrial cancer were not considered overweight. Thirteen of the 20 patients were diagnosed either with a premalignant lesion, such as endometrial hyperplasia with atypia or early invasive disease such as a well-differentiated carcinoma developing in an endometrial polyp but without invasion. In five of these cases, patients were asymptomatic and the diagnosis of malignancy was established only as an incidental finding after hysterectomy and vaginal repair for pelvic relaxation.

Severe cardiac disease was present in three patients. In one patient with liver metastases from colon cancer the hysterectomy was also performed vaginally.

The median operative time was $1 \mathrm{~h}$ and $44 \mathrm{~min}$, with a median blood loss of $250 \mathrm{cc}$ (Table 3). Both operative time and blood loss were significantly correlated with the patients' weight. 
Table 3. Surgery

\begin{tabular}{llc}
\hline Median operative time & \multicolumn{2}{l}{$104 \mathrm{~min}(50-170 \mathrm{~min})$} \\
Median intraoperative blood loss $\dagger$ & $250 \mathrm{cc}(100-1200 \mathrm{cc})$ \\
Additional surgical procedures & $n$ & $\%$ \\
Anterior/posterior repair & 10 & 17 \\
Bilateral salpingo-oophorectomy & 7 & 12 \\
Unilateral salpingo-oophorectomy & 6 & 10 \\
Morcellation & 4 & 7 \\
Schuchardt incision & 2 & 3 \\
\hline
\end{tabular}

*Correlated with patients' weight: $r=0.56 ; P=0.004$.

$\dagger$ Correlated with patients' weight: $r=0.46 ; P=0.011$.

An anterior and posterior repair was done in 10 cases $(17 \%)$. Removal of both ovaries and tubes was attempted in all patients, but was feasible in only seven cases for technical reasons. In another six cases, only a unilateral salpingo-oophorectomy could be performed. Morcellation and the Schuchardt incision were used in four and two cases, respectively.

The complication rate was low (Table 4). None of the 60 hysterectomies had to be completed abdominally. Intraoperative bleeding requiring transfusion was encountered in four patients $(7 \%)$ and vaginal cuff cellulitis occurred in four patients. There were no bladder or rectal injuries and no pulmonary embolization occurred. Operative mortality was $0 \%$. Hospital stay was short, with a median duration of 6 days and a range of 2-16 days.

Table 4. Complications

\begin{tabular}{lll}
\hline & $n$ & $\%$ \\
\hline Bleeding requiring transfusion & 4 & 7 \\
$\begin{array}{l}\text { Postoperative infection } \\
\text { (vaginal cuff cellulitis) }\end{array}$ & 4 & 7 \\
\hline
\end{tabular}

Histologic evaluation showed $90 \%$ of cancers to be adenocarcinomas (Fig. 1). Three of the patients with adenocarcinomas also had squamous differentiation (5.0\%), one had an adenosquamous lesion, one a clear cell carcinoma and one a carcinosarcoma. The majority of tumors were well differentiated $(68 \%) ; 18 \%$ were

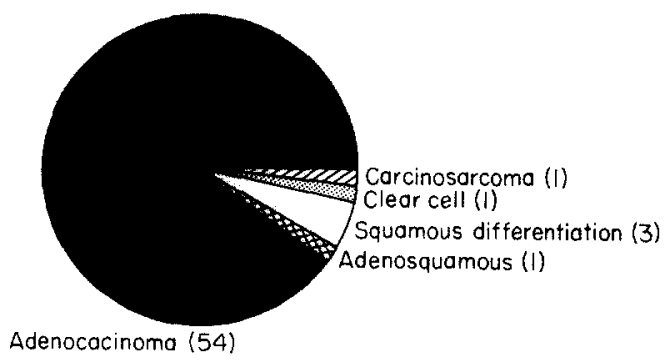

Fig. 1. Histologic type. $n=60$. moderately differentiated; and $7 \%$ poorly differentiated (Fig. 2). In four instances, pathology slides were not available for review; however, all had documented invasive disease.

In $41 \%$, there was no myometrial invasion (Fig. 3). In $43 \%$ of the specimens there was invasion to the inner one-third of the myometrial thickness. Cervical involvement was present in three patients. In no case was there serosal involvement of the uterus.

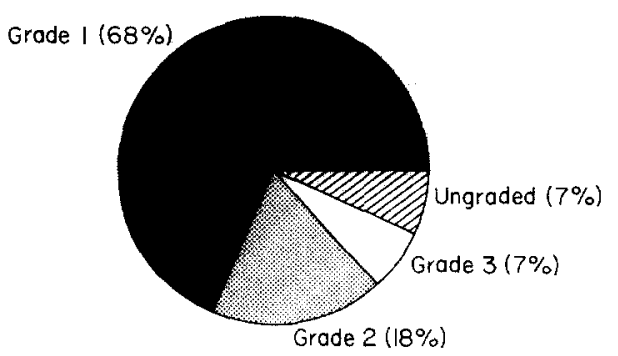

Fig. 2. Grade of differentiation. $n=60$.

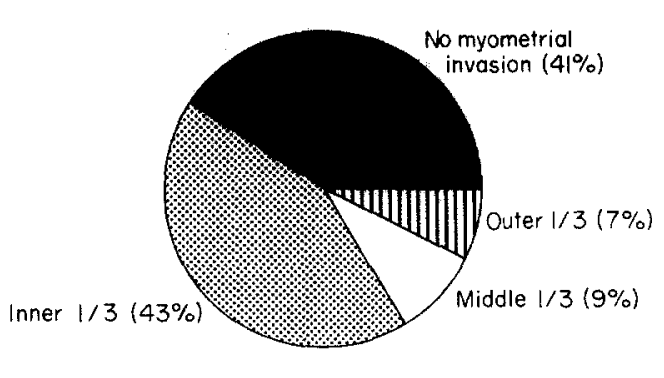

(cervical involvement in three patients)

Fig. 3. Depth of invasion. $n=60$.

Preoperative radiation therapy, usually consisting of an intracavitary radium or cesium implant, was given to 15 patients (Table 5). All 15 patients showed residual disease in the uterine specimen. Preoperative radiation was not given after 1981. Postoperative radiation therapy was used in combination with the surgery in nine patients.

The results of follow-up are summarized in Table 6 . The median follow-up time was 7 years. Forty-one out

Table 5. Adjuvant radiation therapy

\begin{tabular}{lrr}
\hline & $n$ & $\%$ \\
\hline Preoperative radiation & 15 & 25.0 \\
Brachytherapy & 14 & 23.3 \\
External radiation & 1 & 1.7 \\
& & \\
Postoperative radiation & 9 & 15.0 \\
Brachytherapy & 4 & 6.7 \\
External radiation & 2 & 3.3 \\
Both & 3 & 5.0 \\
\hline
\end{tabular}


Table 6. Outcome*

\begin{tabular}{lcc}
\hline & $n$ & $\%$ \\
\hline Diagnosis at least 5 years ago & 45 & 100 \\
Alive NED at 5 years & 41 & 91.1 \\
Dead NED at 5 years & 4 & 8.9 \\
DOD at 5 years & 0 & 0 \\
Diagnosis at least 10 years ago & 31 & 100 \\
Alive NED at 10 years & 27 & 87.1 \\
Dead NED at 10 years & 2 & 6.5 \\
No information at 10 years & 1 & 3.2 \\
DOD at 10 years & 1 & 3.2 \\
\hline
\end{tabular}

"Median follow-up 7 years (1-24 years). NED, no evidence of disease; DOD, died of disease.

of 45 patients were alive at 5 years $(91.1 \%)$, and 27 out of $31(87.1 \%)$ at 10 years. Only one patient developed recurrence and subsequently died from metastatic disease. Briefly, this patient was a 73-year-old white female who underwent vaginal hysterectomy because of her morbid obesity ( 250 pounds) in combination with hypertension and cardiac disease (aortic stenosis). She received a preoperative cesium application. Histologic evaluation showed a grade 3 adenosquamous carcinoma with $60 \%$ myometrial invasion and extension to the cervix. The patient then received 40 Gy of external beam radiation to the whole pelvis. At 5 years she was without evidence of disease. Six years and 3 months after the initial surgery she died from lung metastases, presumably arising from the endometrial cancer. No pelvic recurrence was documented at that time. Unfortunately, no autopsy was performed.

\section{Discussion}

A GOG study of 621 patients with clinical stage I carcinoma of the endometrium clearly demonstrated that an appreciable number of early stage patients (22\%) had disease outside the uterus, with spread to pelvic and/or para-aortic lymph nodes, adnexal disease, intraperitoneal spread or positive pelvic washings ${ }^{(8)}$. This disease spread cannot be evaluated effectively with our present diagnostic tools, such as lymphangiography, CT scan or MRI. The most accurate assessment is achieved by surgical staging, which requires a laparotomy and removal of the uterus and the adnexa, pelvic washings and selective lymphadenectomy from the pelvic and para-aortic area. As endometrial cancer is frequently associated with older age, morbid obesity, diabetes mellitus, cardiac disease, hypertension, and other risk factors, not all patients are candidates for retroperitoneal node dissections or even abdominal surgery.
An alternative approach would be radiation therapy alone. However, prognosis is not as good as after surgical treatment, as discussed below.

A hysterectomy using the vaginal approach seems to be a suitable alternative, as it is well tolerated with few postoperative complications and a low mortality rate. It is as feasible in obese as in non-obese patients ${ }^{(9)}$. Although the older literature seems to support this view $^{(2,10)}$, only part of it holds true today, as anesthesia techniques and postoperative care have been improved significantly. In the past, vaginal hysterectomy for endometrial cancer was justified in all patients with high surgical risk. Today, a low risk of recurrence is also required if this kind of surgery is to be performed ${ }^{(11)}$. Although surgical staging provides the best estimate of the risk of recurrence, some information can be obtained preoperatively. For example, the frequency of nodal metastases in the para-aortic nodes is significantly related to clinical stage, histologic type and grade ${ }^{(8)}$, all of which can be determined prior to hysterectomy.

In our study, all patients had clinical stage I disease. However, three patients had cervical involvement not detected by fractional D\&C. The majority of cases were adenocarcinomas, with only one adenosquamous carcinoma, the latter having an increased risk of developing para-aortic lymph node metastases; and $86 \%$ were either well or moderately differentiated.

Another classical indication for the vaginal approach is pelvic relaxation or prolapse with the necessity of an anterior and/or posterior repair. This was done on 10 patients in our series. In a patient with endometrial cancer, this is acceptable only if the patient belongs to the low-risk group.

Nulliparity and previous gynecological surgery are not absolute contraindications against the vaginal approach. With a large uterus which is located high in the pelvis, a Schuchardt incision facilitates the surgical access. This incision, originally developed by Schuchardt for the vaginal radical hysterectomy in cervical cancer ${ }^{(12)}$, is an incision similar to a mediolateral episiotomy. However, the levator ani muscle is almost completely transected.

Morcellation was done in four patients. Morcellation should be avoided if possible, because of the potential spread of tumor cells upon opening the endometrial cavity. However, none of the four patients in this study appeared to be adversely effected by morcellation, as none of the patients died of disease. Three out of four patients survived more than 5 years. In one patient, the follow-up interval was less than 5 years.

Although adjuvant radiation therapy has not been shown to improve significantly survival from 
endometrial cancer ${ }^{(13)}$, and most patients with clinical stage I disease have a good prognosis anyway, radiation was chosen for patients who were considered to be at high risk for recurrence because of poor tumor differentiation or deep myometrial invasion. Until 1981, patients received preoperative radiation therapy. Interestingly, all of these patients had residual tumor in the uterine specimen at the time of vaginal hysterectomy 4-6 weeks later. After 1981, preoperative radiation was abandoned in favor of the more individualized postoperative radiation therapy.

A complete long-term follow-up for the 60 patients reported in this study is available. Forty-five patients were followed for at least 5 years and 31 patients for at least 10 years. Four patients had died at 5 years and two patients at 10 years of causes other than endometrial cancer. Only one patient with an advanced high-grade adenosquamous carcinoma died of recurrence six years after vaginal hysterectomy for endometrial cancer.

The present study is retrospective and no direct comparisons are possible between the treatment results of vaginal and abdominal surgery or primary radiation therapy at the same institution. Several studies exist, however, that have made an attempt to retrospectively compare abdominal and vaginal hysterectomy as treatment for endometrial cancer. In those institutions, the indication for vaginal surgery was far less restricted than in our study. Scarselli et al. ${ }^{(14)}$ compared two consecutive time intervals. From 1977 to 1979 , the treatment protocol recommended vaginal hysterectomy, bilateral salpingooophorectomy and upper colpectomy. Since 1980, the abdominal route (surgical staging including a selective pelvic but not para-aortic lymphadenectomy) prevailed. There was no significant difference in survival between the two groups ( $87 \%$ versus $88 \%$, respectively). The rate of severe complications was $6.8 \%$ for abdominal surgery and only $1.1 \%$ for vaginal surgery. No operative mortality was observed for patients operated by the vaginal route, but $2.7 \%$ for those operated by the abdominal route.

Candiani et al. ${ }^{(15)}$ compared total abdominal hysterectomy with bilateral salpingo-oophorectomy with and without selective pelvic lymphadenectomy with vaginal hysterectomy and found survival rates of $73 \%, 79 \%$ and $76 \%$, respectively. Similar results had already been reported by the same author in $1978^{(16)}$.

Bloss et al. ${ }^{(17)}$ reported on their experience with vaginal hysterectomy in stage I endometrial cancer of 31 medically compromised patients. The incidence of morbid obesity $(87 \%)$, hypertension $(58 \%)$, diabetes mellitus ( $35 \%)$ and cardiovascular disease $(26 \%)$ is comparable to the present study. The complication rate was also similar (13\%). Three-year disease-free survival was $100 \%$.

Carenza et al. ${ }^{(11)}$ treated 160 patients with vaginal hysterectomy. Sixty-two per cent were obese. The 5year survival in (clinical) stage I endometrial carcinoma was $85.5 \%$, being related to histologic grade (grade 1: $95 \%$; grade $2: 86.7 \%$; grade 3: $63.3 \%$ ).

The studies mentioned above demonstrate that the overall prognosis of clinical stage I endometrial cancer is acceptable as long as the uterus is completely removed. The survival rates after surgery are still at least $15-20 \%$ higher than after radiation therapy ${ }^{(2,3,18)}$.

In conclusion, results of treatment of endometrial cancer achieved by vaginal hysterectomy are favorable if patients are carefully selected. Therefore, vaginal hysterectomy can be an intermediate choice between abdominal hysterectomy and primary radiation therapy in patients with medical contraindications to conventional therapy. Although we consider surgical staging as the standard of care for the treatment of endometrial cancer, vaginal hysterectomy has a definite place in the management of patients with good prognostic criteria who are at high operative risk for the standard surgical approach.

\section{Acknowledgements}

This paper was presented at the Society of Pelvic Surgeons 42nd Annual Meeting in Munich, Germany, 15 September 1992.

\section{References}

1 Van Bouwidijk Bastiannse MA. Cancer of the body of the uterus. Br J Obstet Gynaecol 1952; 59: 611-20.

2 Fauvet E, Meyer-Venter B, Majewski A. Über die Behandlungsergebnisse beim Korpuskarzinom. Geburtsh Frauenheilk 1968; 28:1-10.

3 Knak J, Macheleidt E. Die vaginale Hysterektomie beim Korphuskarzinom. Zbl Gynákol 1981; 103: 271-6.

4 Pratt JH, Symmonds RE, Welch JJ. Vaginal hysterectomy for carcinoma of the fundus. Am J Obstet Gynecol 1964; 88: 1063-71.

5 Park RC, Grigsby PW, Muss HB, Norris HJ. Corpus: epithelial tumors. In: Hoskins WJ, Perez CA, Young RC, eds. Principles and Practice of Gynecologic Oncology. Philadelphia: JB Lippincott, 1992; 663-93.

6 Peters WA, Andersen WA, Thornton WN, Morley GW. The selective use of vaginal hysterectomy in the management of adenocarcinoma of the endometrium. Am J Obstet Gynecol 1983; 146: 285-91.

7 Shepherd JH. Revised FIGO staging for gynaecological cancer. Br J Obstet Gynaecol 1989; 96: 889 
8 Creasman WT, Morrow CP, Bundy BN, Homesley HD, Graham JE, Heller PB. Surgical pathologic spread patterns of endometrial cancer-a Gynecologic Oncology Group Study. Cancer 1987; 60: 2035-41.

9 Pitkin RM. Vaginal hysterectomy in obese women. Obstet Gynecol 1977; 49: 567.

10 Hilfrich $H$ J. Die Komplikationsrate bei den verschiedenen Therapieformen des Korpuskarzinoms. Arch Gynakkol 1973; 214: 284-5.

11 Carenza L, Villani C, Nobili F, Porpora MG, Lukic A, Falqui $\mathrm{L}$. Does today's vaginal surgery still have specific role in the treatment of endometrial cancer? NY Acad Sci 1991; 622: 477-84.

12 Schuchardt K. Eine neue Methode der Gebärmutterexstirpation. Zbl Chir 1983; 1131.

13 Piver MS, Yazigi R, Blumenson L, Tsukada Y. A prospective trial comparing hysterectomy, hysterectomy plus vaginal radium, and uterine radium plus hysterectomy in stage I endometrial carcinoma. Obstet Gynecol 1979; 54: 85.
14 Scarselli G, Savino L, Ceccherini R, Barciulli F, Massi GB. Role of vaginal surgery in the 1st stage endometrial cancer. Eur J Gymaecol Oncol 1992; 8: 15-19.

15 Candiani GB, Belloni C, Maggi R, Colombo G, Frigoli A, Carinelli SG. Evaluation of different surgical approaches in the treatment of endometrial cancer at FIGO stage I. Gynecol Oncol 1990; 37: 6-8.

16 Candiani GB, Mangioni C, Marzi MM. Surgery in endometrial cancer: age, route and operability rate in 854 stage I and II fresh consecutive cases: 1955-1976. Gynecol Oncol 1978; 6: 363-72.

17 Bloss JD, Berman ML, Bloss LP, Buller RE. Use of vaginal hysterectomy for the management of stage I endometrial cancer in the medically compromised patient. Gynecol Oncol 1991; 40: 74-7.

18 Börner P, Heidenreich W, Majewski A. Zunehmende Individualisierung der Therapie des Endometriumkarzinoms. Geburtsh Frauenheilkd 1977; 37: 142-9.

Accepted for publication 14 December 1993 